\title{
Nearly Gorenstein cyclic quotient singularities
}

\section{Alessio Caminata ${ }^{1}(\mathbb{0}) \cdot$ Francesco Strazzanti $^{2}$}

Received: 21 July 2020 / Accepted: 18 September 2020 / Published online: 18 October 2020

(c) The Author(s) 2020

\begin{abstract}
We investigate the nearly Gorenstein property among $d$-dimensional cyclic quotient singularities $\mathbb{k} \llbracket x_{1}, \ldots, x_{d} \rrbracket^{G}$, where $\mathbb{k}$ is an algebraically closed field and $G \subseteq \mathrm{GL}(d, \mathbb{k})$ is a finite small cyclic group whose order is invertible in $\mathbb{k}$. We prove a necessary and sufficient condition to be nearly Gorenstein that also allows us to find several new classes of such rings.
\end{abstract}

Keywords Nearly Gorenstein · Invariant ring · Quotient singularity · Trace ideal

Mathematics Subject Classification 13A50 $\cdot 13 \mathrm{H} 10 \cdot 14 \mathrm{~L} 30$

\section{Introduction}

Gorenstein rings are among the most important objects in commutative algebra and appear in several contexts. On the other hand, despite their celebrated ubiquity (Bass 1963; Huneke 1999), the class of Gorenstein rings is not so large, compared for instance with that of Cohen-Macaulay rings. In many significant cases one encounters CohenMacaulay rings which are not Gorenstein rings. For this reason, many researchers started looking for generalizations of the notion of Gorenstein aiming to find a class of Cohen-Macaulay rings which is still able to capture some of the interesting properties

The second author was partially supported by INdAM, more precisely he was "titolare di una borsa per l'estero dell'Istituto Nazionale di Alta Matematica" and "titolare di un Assegno di Ricerca dell'Istituto Nazionale di Alta Matematica".

$\triangle \quad$ Alessio Caminata

alessio.caminata@unine.ch

Francesco Strazzanti

francesco.strazzanti@unibo.it

1 Institut de Mathématiques, Université de Neuchâtel, Rue Emile-Argand 11, 2000 Neuchâtel, Switzerland

2 Dipartimento di Matematica, Università di Bologna, Piazza di Porta San Donato 5, 40126 Bologna, Italy 
of Gorenstein rings. In recent years, two of these new classes of rings have drawn particular attention: almost Gorenstein and nearly Gorenstein rings. Almost Gorenstein rings were first defined by Barucci and Fröberg (1997) for one-dimensional analytically unramified rings and later generalized by Goto and Matsuoka (2013); Goto et al. (2015). This notion has already been largely investigated and many properties are known, see for example Endo et al. (2020), Goto et al. (2016), Herzog et al. (2019), Taniguchi (2018) and the references therein. On the other hand, nearly Gorenstein rings, which are the object of interest of this paper, have been introduced even more recently by Herzog et al. (2019) in 2019, although their defining property was already examined by Ding (1993), Huneke and Vraciu (2006), and Striuli and Vraciu (2011). Moreover, nearly Gorenstein rings have been studied in several contexts, such as zero-dimensional schemes (Kreuzer et al. 2019), affine semigroup rings Herzog et al. (2019), and affine monomial curves Moscariello (2020). See also Endo et al. (2020), Dao et al. (2020), Kobayashi (2020), Kumashiro (2020), Rahimi (2020) for other related results.

To explain the definition and the motivation of nearly Gorenstein rings we start with a Cohen-Macaulay local ring $(R, \mathfrak{m})$ which admits a canonical module $\omega_{R}$. The trace of the canonical module, denoted by $\operatorname{tr}\left(\omega_{R}\right)$, is defined as the sum of the ideals $\varphi\left(\omega_{R}\right)$, where the sum is taken over all the $R$-module homomorphisms $\varphi: \omega_{R} \rightarrow R$. The importance of $\operatorname{tr}\left(\omega_{R}\right)$ comes from the fact that it describes the non-Gorenstein locus of $R$, since the localization $R_{\mathfrak{p}}$ at a prime ideal $\mathfrak{p}$ is not Gorenstein if and only if $\operatorname{tr}\left(\omega_{R}\right) \subseteq \mathfrak{p}$. In particular, it follows that $R$ is Gorenstein if and only if $\operatorname{tr}\left(\omega_{R}\right)=R$. For this reason, one defines $R$ to be nearly Gorenstein when $\mathfrak{m} \subseteq \operatorname{tr}\left(\omega_{R}\right)$. It is now clear that a nearly Gorenstein ring is Gorenstein on the punctured spectrum, but the converse does not occur in general. Moreover, it also holds that a one-dimensional almost Gorenstein ring is nearly Gorenstein, even though this is no longer true in the higher dimensional case, where the relation between these two notions remains unclear.

In this paper, we look at the nearly Gorenstein property for quotient singularities. Let $R=\mathbb{k} \llbracket x_{1}, \ldots, x_{d} \rrbracket$ be a $d$-dimensional formal power series ring over an algebraically closed field $\mathbb{k}$ and let $G$ be a finite subgroup of $\operatorname{GL}(d, \mathbb{k})$ acting linearly on $R$. The corresponding invariant ring $R^{G}$ is the completion at the origin of the coordinate ring of the quotient variety $\mathbb{A}_{\mathbb{k}}^{d} / G$, so we will refer to it as a quotient singularity. The study of these objects and their properties lies at the intersection of several branches of mathematics and has been largely explored both from a geometric and an algebraic point of view. In the modular case, i.e., when the characteristic of $\mathbb{k}$ divides the order of the group $G$, even the Cohen-Macaulay property is not fully understood (see e.g. (Campbell and Wehlau 2011, Example 8.0.9) or Kemper 1999), so we will rather focus on the non-modular situation, that is when char $\mathbb{k} \nmid|G|$. Under this assumption it is well known that the invariant ring $R^{G}$ is a complete local normal domain and it is Cohen-Macaulay thanks to Hochster-Eagon's Theorem (Hochster and Eagon 1971). Moreover, thanks to an old result of Prill (1967) it is not restrictive to assume further that the acting group is small, i.e., it does not contain pseudo-reflections. In this case, by a result of Watanabe (1974a, b) the Gorenstein property of these rings is also well understood. Namely, $R^{G}$ is Gorenstein if and only if the group $G$ is contained in $\operatorname{SL}(d, \mathbb{k})$. Therefore, it arises as a natural problem to look for a characterization of 
the nearly Gorenstein property for these rings. In fact, we investigate precisely this question for an important class of quotient singularities: cyclic quotient singularities, i.e., when the group $G$ is cyclic.

For this class, we are able to find a numerical criterion which gives a necessary and sufficient condition for the ring $R^{G}$ to be nearly Gorenstein. Using this criterion, we identify several families of nearly Gorenstein rings. We recall that if $G$ is a cyclic small subgroup of $\operatorname{GL}(d, \mathbb{k})$ of order $n$ with char $\mathbb{k} \nmid n$, we can assume that it is generated by a diagonal matrix $\phi=\operatorname{diag}\left(\lambda^{t_{1}}, \ldots, \lambda^{t_{d}}\right)$, where $\lambda$ is a primitive $n$-th root of unity in $\mathbb{k}$ and $t_{1}, \ldots, t_{d}$ are positive integers such that $\operatorname{gcd}\left(t_{i_{1}}, \ldots, t_{i_{d-1}}, n\right)=1$ for every $(d-1)$-tuple with distinct integers $i_{1}, \ldots, i_{d-1} \in\{1, \ldots, d\}$. We denote the corresponding invariant ring $R^{G}$ by $\frac{1}{n}\left(t_{1}, \ldots, t_{d}\right)$.

Theorem A (see Proposition 2.4 and Corollaries 2.5, 2.6, 2.9) Let $n, d \geq 2$ and $t_{1}, \ldots, t_{d} \geq 1$ be integers and assume that at least one of the following holds:

- $d=2$;

- $n \leq 3$;

- $t_{1} \equiv \cdots \equiv t_{d} \equiv 1 \bmod n$;

- $t_{1} \equiv \cdots \equiv t_{d-1} \equiv 1 \bmod n$ and $t_{d} \equiv-d+2 \bmod n$.

Then, the cyclic quotient singularity $\frac{1}{n}\left(t_{1}, \ldots, t_{d}\right)$ is nearly Gorenstein.

In the case $t_{1} \equiv \cdots \equiv t_{d} \equiv 1 \bmod n$ the corresponding invariant ring is a Veronese subalgebra of $R$. We also notice that, when $R$ is a Gorenstein positively graded $\mathbb{k}$-algebra with positive dimension, Veronese subalgebras of $R$ are known to be nearly Gorenstein by (Herzog et al. 2019), Corollary 4.7. Moreover, Theorem A says that if the dimension is two or if the order of the group is at most 3 , then cyclic quotient singularities are always nearly Gorenstein. However, as soon as these assumptions are dropped we may find examples of cyclic quotient singularities that are not nearly Gorenstein. For instance, the invariant ring $\frac{1}{4}(1,2,3)$ is not nearly Gorenstein (see Example 2.8). More generally, the numerical criterion we proved can be implemented to find all nearly Gorenstein cyclic quotient singularities for some values of $n$ and $d$. For example, see Table 1 for an exhaustive list of non-isomorphic nearly Gorenstein cyclic quotient singularities with small values of $n$ and $d$.

In order to measure the distance of a quotient singularity $R^{G}$ to be Gorenstein or nearly Gorenstein, one can consider its residue which is the length $\operatorname{res}\left(R^{G}\right)=$ $\ell_{R^{G}}\left(R^{G} / \operatorname{tr}\left(\omega_{R^{G}}\right)\right)$. We have that $\operatorname{res}\left(R^{G}\right)=0$ if and only if $R^{G}$ is Gorenstein, and $\operatorname{res}\left(R^{G}\right)=1$ precisely when $R^{G}$ is nearly Gorenstein, but not Gorenstein. Thus, from Theorem A follows that every two-dimensional cyclic quotient singularity $R^{G}$ has $\operatorname{res}\left(R^{G}\right) \leq 1$. However, already in dimension 3 we are able to produce cyclic quotient singularities of arbitrarily large residue.

Theorem B (see Theorem 2.10) Let $n$ and $m$ be two coprime positive integers with $n \geq 3$ and $m<\left\lceil\frac{n}{2}\right\rceil$. Then the cyclic quotient singularity $\frac{1}{n}(1, m, n-1)$ has residue $m$.

In Sect. 3 we consider the field of complex numbers $\mathbb{C}$ and we turn our attention to the two-dimensional case, where the finite small group $G \subseteq \operatorname{GL}(2, \mathbb{C})$ is 
not necessarily cyclic. The nearly Gorenstein property of the corresponding invariant rings was studied by Ding, who gave a complete classification of nearly Gorenstein two-dimensional quotient singularities (see Ding 1993, Proposition 3.5). However, somehow a case was left out of Ding's classification: it is the invariant ring of the octahedral group $\mathbb{O}_{11}$ obtained by adding to the binary octahedral subgroup of $\operatorname{SL}(2, \mathbb{C})$ a cyclic generator of the form $\operatorname{diag}(\lambda, \lambda)$, where $\lambda \in \mathbb{C}$ is a primitive root of unity of order 22. We prove that this ring is nearly Gorenstein in Proposition 3.1.

The structure of the paper is the following. First, in Sect. 1 we review some basic definitions and notations on nearly Gorenstein rings and quotient singularities. Then, in Sect. 2 we focus on nearly Gorenstein cyclic quotient singularities. In Theorem 2.3 we prove a numerical criterion that characterizes them and we use this to provide several classes of nearly Gorenstein rings as stated in Theorem A. Finally, in Sect. 3 we study the nearly Gorenstein octahedral singularity mentioned above which completes Ding's classification.

\section{Preliminaries}

In this section we recall some basic definitions and standard facts on nearly Gorenstein rings and quotient singularities.

\subsection{Nearly Gorenstein rings}

Let $(R, \mathfrak{m})$ be a Cohen-Macaulay local ring which admits a canonical module $\omega_{R}$. The trace of the canonical module, denoted by $\operatorname{tr}\left(\omega_{R}\right)$, is the sum of the ideals $\varphi\left(\omega_{R}\right)$ for any $R$-module homomorphism $\varphi: \omega_{R} \rightarrow R$. In other words, we have

$$
\operatorname{tr}\left(\omega_{R}\right)=\sum_{\varphi \in \operatorname{Hom}_{R}\left(\omega_{R}, R\right)} \varphi\left(\omega_{R}\right)
$$

The trace of $\omega_{R}$ describes the non-Gorenstein locus of $R$. In fact, given a prime ideal $\mathfrak{p} \subseteq R$, then $R_{\mathfrak{p}}$ is not Gorenstein if and only if $\operatorname{tr}\left(\omega_{R}\right) \subseteq \mathfrak{p}$ (cf. Herzog et al. 2019, Lemma 2.1). In particular, since $\operatorname{tr}\left(\omega_{R}\right)$ is an ideal, one has that $R$ is Gorenstein if and only if $\operatorname{tr}\left(\omega_{R}\right)=R$.

Definition 1.1 (Herzog et al. 2019). $R$ is called nearly Gorenstein if $\mathfrak{m} \subseteq \operatorname{tr}\left(\omega_{R}\right)$.

It is immediately clear from the definition that Gorenstein rings are nearly Gorenstein and that $R$ is nearly Gorenstein but not Gorenstein if and only if $\operatorname{tr}\left(\omega_{R}\right)=\mathfrak{m}$. In order to give a measure to the distance of a ring to be Gorenstein or nearly Gorenstein, one defines the residue of $R$ as

$$
\operatorname{res}(R)=\ell_{R}\left(R / \operatorname{tr}\left(\omega_{R}\right)\right) \in \mathbb{N} \cup\{\infty\} .
$$

The ring $R$ is Gorenstein if and only if $\operatorname{res}(R)=0$ and it is nearly Gorenstein if and only if $\operatorname{res}(R) \leq 1$. 
If there exists a canonical module $\omega_{R}$ that is also an ideal of $R$ we say that $\omega_{R}$ is a canonical ideal of $R$. In this case there is a useful formula to find its trace. We denote the total ring of fractions of $R$ by $Q(R)$.

Lemma 1.2 (Herzog et al. 2019, Lemma 1.1) Let $(R, \mathfrak{m})$ be a local domain with a canonical ideal $\omega_{R}$. Then, the trace ideal of the canonical module of $R$ is equal to $\operatorname{tr}\left(\omega_{R}\right)=\omega_{R}\left(R: Q(R) \omega_{R}\right)$.

In particular, if $\omega_{R}$ is a canonical ideal, then it is included in $\operatorname{tr}\left(\omega_{R}\right)$ because $1 \in$ $\left(R: Q(R) \omega_{R}\right)$.

\subsection{Quotient singularities}

Let $\mathbb{k}$ be an algebraically closed field and let $G$ be a finite subgroup of $\operatorname{GL}(d, \mathbb{k})$ such that the order $|G|$ of $G$ is coprime with the characteristic of $\mathbb{k}$. We consider a power series ring $R=\mathbb{k} \llbracket x_{1}, \ldots, x_{d} \rrbracket$ over $\mathbb{k}$. The group $G$ acts linearly on $R$ with the action on the variables $x_{1}, \ldots, x_{d}$ given by matrix multiplication. We denote by $R^{G}$ the ring of invariants under this action and we will call it also (non-modular) quotient singularity.

We recall that an element $\sigma \in G$ is called pseudo-reflection if the fixed subspace $\left\{v \in \mathbb{k}^{d}: \sigma v=v\right\}$ has dimension $d-1$. We will always assume that the acting group $G$ is small, i.e., that it does not contain pseudo-reflections. This is not restrictive in our setting. In fact, by a theorem of Prill (1967) if $G$ is not small we can replace $R$ by another power series ring $S$ and find a small finite linear group $H$ such that $R^{G} \cong S^{H}$. This is essentially a consequence of the Chevalley-Shephard-Todd Theorem which implies that the ring of invariants of a finite group generated by pseudo-reflections acting on a power series ring is again a regular local ring.

Under the previous assumptions, the quotient singularity $R^{G}$ is a Cohen-Macaulay complete local normal domain of dimension $d$. Watanabe $(1974 \mathrm{a}, \mathrm{b})$ proved that $R^{G}$ is Gorenstein if and only if $G \subseteq \operatorname{SL}(d, \mathbb{k})$. In this case $R^{G}$ is called special quotient singularity. If $G$ is a cyclic group, then $R^{G}$ is called cyclic quotient singularity. The Kleinian singularities are the two-dimensional complex special quotient singularities $\mathbb{C} \llbracket x_{1}, x_{2} \rrbracket^{G}$.

In order to study the nearly Gorenstein property of quotient singularities, it is important to understand their canonical module. To this purpose, we introduce the following definition.

Definition 1.3 Let $R=\mathbb{k} \llbracket x_{1}, \ldots, x_{d} \rrbracket$ be a power series ring and let $G$ be a finite small subgroup of $\operatorname{GL}(d, \mathbb{k})$ such that char $\mathbb{k} \nmid|G|$. We say that an element $f \in R$ is a $G$-canonical element of $R$ if $\sigma(f)=\operatorname{det} \sigma \cdot f$ for all $\sigma \in G$.

The previous definition is motivated by the following result due to Singh (1970) and Watanabe (Watanabe 1974b, Theorem 1') (see also (Richard 1978) or (Bruns and Herzog 1998, Theorem 6.4.9) for an alternative proof).

Theorem 1.4 (Singh-Watanabe) Let $R$ and $G$ be as above and let $f \in R$ be a $G$ canonical element, then $f R \cap R^{G}$ is a canonical ideal of $R^{G}$. 


\section{Cyclic quotient singularities}

In this section we focus on the nearly Gorenstein property for cyclic quotient singularities. We consider a formal power series ring $R=\mathbb{k} \llbracket x_{1}, \ldots, x_{d} \rrbracket$ over an algebraically closed field $\mathbb{k}$ and a finite small cyclic group $G \subseteq \operatorname{GL}(d, \mathbb{k})$ such that $|G|=n$ is not zero in $\mathbb{k}$.

Since $G$ is a finite cyclic group, we can assume that it is generated by a diagonal matrix $\phi$ of the form

$$
\phi=\operatorname{diag}\left(\lambda^{t_{1}}, \ldots, \lambda^{t_{d}}\right)=\left(\begin{array}{cccc}
\lambda^{t_{1}} & 0 & \ldots & 0 \\
0 & \lambda^{t_{2}} & \ldots & 0 \\
\vdots & \vdots & \ddots & \vdots \\
0 & 0 & \ldots & \lambda^{t_{d}}
\end{array}\right)
$$

where $\lambda$ is a primitive $n$-th root of unit in $\mathbb{k}$ and $t_{1}, \ldots, t_{d} \geq 0$ are integers. If $t_{d} \equiv 0$ $\bmod n$, one can set $S=\mathbb{k} \llbracket x_{1}, \ldots, x_{d-1} \rrbracket$ and $H \subseteq \mathrm{GL}(d-1, \mathbb{k})$ the group generated by $\operatorname{diag}\left(\lambda^{t_{1}}, \ldots, \lambda^{t_{d-1}}\right)$, then $R^{G}$ is nearly Gorenstein if and only if $S^{H}$ is Gorenstein, by (Herzog et al. 2019, Proposition 4.5). For this reason, we will assume without loss of generality that $t_{1}, \ldots, t_{d} \not \equiv 0 \bmod n$. In this case, the lack of pseudo-reflections in $G$ is equivalent to the condition $\operatorname{gcd}\left(t_{i_{1}}, \ldots, t_{i_{d-1}}, n\right)=1$ for every $(d-1)$-tuple with distinct integers $i_{1}, \ldots, i_{d-1} \in\{1, \ldots, d\}$. With these conventions, we denote the cyclic quotient singularity $R^{G}$ by $\frac{1}{n}\left(t_{1}, \ldots, t_{d}\right)$. We point out that this notation is not unique. For instance $\frac{1}{3}(1,1,2)=\frac{1}{3}(2,2,1)$ are equal because they are invariant rings with respect to the same group. Since the action of $G$ on $R$ is diagonal, the $\mathbb{k}$ algebra $\frac{1}{n}\left(t_{1}, \ldots, t_{d}\right)$ can be generated by monomials, more precisely one can choose a (non-minimal) system of generators as follows

$$
R^{G}=\mathbb{k} \llbracket x_{1}^{\alpha_{1}} \ldots x_{d}^{\alpha_{d}} \mid \alpha_{1}+\cdots+\alpha_{d} \leq n \text { and } \alpha_{1} t_{1}+\cdots+\alpha_{d} t_{d} \equiv 0 \quad \bmod n \rrbracket .
$$

Proposition 2.1 Let $R$ and $G$ be as above. A $G$-canonical element of $R$ is given by $f=x_{1} x_{2} \ldots x_{d}$. Moreover, we have

$$
\operatorname{tr}\left(\omega_{R^{G}}\right)=(f R)^{G}\left(R:_{Q(R)} f R\right)^{G} .
$$

Proof Since $G$ is generated by $\phi=\operatorname{diag}\left(\lambda^{t_{1}}, \ldots, \lambda^{t_{d}}\right)$, to prove that $f$ is a $G$-canonical element it is enough to observe that $\phi(f)=\lambda^{t_{1}} \ldots \lambda^{t_{d}} f=\operatorname{det}(\phi) f$.

We now prove that $\operatorname{tr}\left(\omega_{R^{G}}\right)=\left(\begin{array}{lll}f R)^{G}(R: Q(R) & f R\end{array}\right)^{G}$. First, notice that by Lemma 1.2 and Theorem 1.4 we have $\operatorname{tr}\left(\omega_{R^{G}}\right)=\left(f R \cap R^{G}\right)\left(R^{G}:_{Q\left(R^{G}\right)}\left(f R \cap R^{G}\right)\right)$. So, since $f R \cap R^{G}=(f R)^{G}$, it is enough to prove that

$$
R^{G}:_{Q\left(R^{G}\right)}\left(f R \cap R^{G}\right)=(R: Q(R) f R)^{G} .
$$

The inclusion $\left(R:_{Q(R)} f R\right)^{G} \subseteq\left(R^{G}:_{Q\left(R^{G}\right)}\left(f R \cap R^{G}\right)\right)$ is clear. Conversely, consider an element $a / b \in\left(R^{G}:_{Q\left(R^{G}\right)}\left(f R \cap R^{G}\right)\right)$ with $\operatorname{gcd}(a, b)=1$. By hypothesis 
$\operatorname{gcd}\left(t_{1}, \ldots, t_{d-1}, n\right)=1$ and so there exist $a_{1}, \ldots, a_{d-1}$ positive integers such that $a_{1} t_{1}+\cdots+a_{d-1} t_{d-1} \equiv 1 \bmod n$. Therefore, there exists a positive $r$ such that

$$
\left(a_{1} t_{1}+\cdots+a_{d-1} t_{d-1}\right) r+t_{1}+\cdots+t_{d-1}+t_{d} \equiv 0 \quad \bmod n .
$$

This implies that $h=\left(x_{1}^{a_{1}} x_{2}^{a_{2}} \ldots x_{d-1}^{a_{d-1}}\right)^{r} f \in R^{G}$, because $\phi(h)=\left(\prod_{i=1}^{d-1} \lambda^{r a_{i} t_{i}}\right.$ $\left.\prod_{i=1}^{d} \lambda^{t_{i}}\right) h=h$. It follows that $a h / b \in R^{G}$, then $b$ is a monomial and $x_{d}^{2}$ does not divide $b$. Since we can repeat the same reasoning with respect to every variable, we get that $b$ is squarefree and, therefore, $b$ divides $f$. This means that $a f / b \in R$ and, then, $a / b \in(R: Q(R) f R)^{G}$ as required.

Lemma 2.2 Let $h=x_{1}^{a_{1}} \ldots x_{d}^{a_{d}}$ be a monomial of $R^{G}$ and let $f=x_{1} x_{2} \ldots x_{d}$. Then, $h \in \operatorname{tr}\left(\omega_{R^{G}}\right)$ if and only if one of the following two conditions holds:

1. $a_{i}>0$ for all $i=0, \ldots, d$;

2. $h=x_{\sigma(1)}^{a_{1}} \cdots x_{\sigma(j)}^{a_{j}}$ with $j<d$, where $\sigma$ is a permutation of $\{1, \ldots, d\}$ and there exist integers $b_{1}, \ldots, b_{j}$ such that $0<b_{k} \leq a_{k}+1$ for every $k \in\{1, \ldots, j\}$ and $\sum_{k=1}^{j} b_{k} t_{\sigma(k)} \equiv-\sum_{k=j+1}^{d} t_{\sigma(k)} \bmod n$.

Proof If $a_{i}>0$ for every $i$, we observe that $h \in f R \subseteq \operatorname{tr}\left(\omega\left(R^{G}\right)\right)$ because $1 \in$ $(R: Q(R) f R)^{G}$. Therefore, without loss of generality we suppose that $h=x_{1}^{a_{1}} \ldots x_{j}^{a_{j}}$ for some $j<d$. We recall that by Proposition $2.1 \operatorname{tr}\left(\omega_{R^{G}}\right)=(f R)^{G}(R: Q(R) f R)^{G}$. Moreover, we observe that $(f R)^{G}$ and $(R: Q(R) f R)^{G}$ are generated by monomials and quotient of monomials respectively because $f$ is a monomial and $G$ is cyclic. Therefore, since $h$ is a monomial, we have that $h \in \operatorname{tr}\left(\omega_{R^{G}}\right)$ if and only if there is an equality

$$
h=\left(x_{1}^{b_{1}} \ldots x_{d}^{b_{d}}\right) \frac{x_{1}^{c_{1}} \ldots x_{d}^{c_{d}}}{x_{1}^{e_{1}} \ldots x_{d}^{e_{d}}}
$$

with $x_{1}^{b_{1}} \ldots x_{d}^{b_{d}} \in(f R)^{G}$ and $x_{1}^{c_{1}} \ldots x_{d}^{c_{d}} / x_{1}^{e_{1}} \ldots x_{d}^{e_{d}} \in(R: Q(R) f R)^{G}$, where we assume that the fraction is irreducible. Since $f=x_{1} \ldots x_{d}$, it follows that $b_{i} \geq 1$ and $e_{i} \leq 1$ for every $i=1, \ldots, d$. Moreover, for every $k=j+1, \ldots, d$ we have $a_{k}=0$ which implies $b_{k}=e_{k}=1$ and $c_{k}=0$. We also note that $a_{i}=b_{i}+c_{i}-e_{i}$ for every $i$ and, therefore, $1 \leq b_{i} \leq a_{i}+1$. Since $h \in R^{G}$, if $x_{1}^{b_{1}} \ldots x_{j}^{b_{j}} x_{j+1} \ldots x_{d}$ is invariant under the action of $G$, also $x_{1}^{c_{1}} \ldots x_{j}^{c_{j}} / x_{1}^{e_{1}} \ldots x_{j}^{e_{j}} x_{j+1} \ldots x_{d}$ is invariant. Recall that $R^{G}=\mathbb{k} \llbracket x_{1}^{\alpha_{1}} \ldots x_{d}^{\alpha_{d}} \mid \alpha_{1}+\cdots+\alpha_{d} \leq n$ and $\alpha_{1} t_{1}+\cdots+\alpha_{d} t_{d} \equiv 0 \bmod n \rrbracket$. Then, it is possible to write $h$ as in (1) if and only if there exist integers $b_{1}, \ldots, b_{j}$ such that $1 \leq b_{i} \leq a_{i}+1$ and $\sum_{i=1}^{j} b_{i} t_{i}+\sum_{k=j+1}^{d} t_{k} \equiv 0 \bmod n$.

Observing that $R^{G}$ is nearly Gorenstein if and only if the conditions of Lemma 2.2 hold for every generator of the maximal ideal of $R^{G}$ we get the following criterion.

Theorem 2.3 The ring $R^{G}$ is nearly Gorenstein if and only if for every $0<i<d$, every permutation $\sigma$ of $\{1, \ldots, d\}$ and every $i$-tuple $\left(a_{1}, \ldots, a_{i}\right)$ of positive integers such that $a_{1}+\cdots+a_{i} \leq n$ and $a_{1} t_{\sigma(1)}+\cdots+a_{i} t_{\sigma(i)} \equiv 0 \bmod n$, there exist integers $b_{1}, \ldots, b_{i}$ such that $\sum_{j=1}^{i} b_{j} t_{\sigma(j)} \equiv-\sum_{k=i+1}^{d} t_{\sigma(k)} \bmod n$ and $0<b_{j} \leq a_{j}+1$ for every $j \in\{1, \ldots, i\}$. 
We want to use the previous theorem to find examples of nearly Gorenstein cyclic quotient singularities. First, we recall that by Watanabe's Theorem a $\frac{1}{n}\left(t_{1}, \ldots, t_{d}\right)$ singularity is Gorenstein if and only if the acting group $G$ is contained in $\operatorname{SL}(d, \mathbb{k})$ which is in turn equivalent to the condition $t_{1}+\cdots+t_{d} \equiv 0 \bmod n$. For instance, for each dimension $d$ the singularity $\frac{1}{n}\left(1, \ldots, 1, t_{d}\right)$ with $t_{d} \equiv-d+1 \bmod n$ is Gorenstein. In a similar fashion, we can obtain examples of nearly Gorenstein cyclic quotient singularities in every dimension.

Proposition 2.4 Let $d \geq 3$ and $n \geq 3$ be integers such that $\operatorname{gcd}(-d+2, n)=1$. Choose an integer $t_{d} \geq 1$ such that $t_{d} \equiv-d+2 \bmod n$. Then, the quotient singularity $\frac{1}{n}\left(1, \ldots, 1, t_{d}\right)$ is nearly Gorenstein, but not Gorenstein.

Proof As usual let $R=\mathbb{k} \llbracket x_{1}, \ldots, x_{d} \rrbracket$ and consider the group $G$ generated by $\operatorname{diag}\left(\lambda, \ldots, \lambda, \lambda^{t_{d}}\right)$ for a primitive $n$-th root of unity $\lambda \in \mathbb{k}$, so that $R^{G}=$ $\frac{1}{n}\left(1, \ldots, 1, t_{d}\right)$. It is clear that $R^{G}$ is not Gorenstein, since $1+\cdots+1-d+2=1 \not \equiv 0$ $\bmod n$. We prove that $R^{G}$ is nearly Gorenstein by using Theorem 2.3. Consider $0<i<d$, a permutation $\sigma$ of $\{1, \ldots, d\}$ and a $i$-tuple $\left(a_{1}, \ldots, a_{i}\right)$ of positive integers such that $a_{1}+\cdots+a_{i} \leq n$ and $a_{1} t_{\sigma(1)}+\cdots+a_{i} t_{\sigma(i)} \equiv 0 \bmod n$. If $t_{\sigma(1)}=\cdots=t_{\sigma(i)}=1$, then we have $a_{1}+\cdots+a_{i}=n$. Therefore, the sum $\sum_{j \leq i} b_{j}$ for $0<b_{j} \leq a_{j}+1$ runs over all possible residues modulo $n$, thus there exist $b_{j}$ 's such that $\sum_{j=1}^{i} b_{j} t_{\sigma(j)} \equiv-\sum_{k=i+1}^{d} t_{\sigma(k)} \bmod n$ is satisfied.

Suppose now that $t_{\sigma(1)}=\cdots=t_{\sigma(i-1)}=1$ and $t_{\sigma(i)}=t_{d} \equiv-d+2 \bmod n$. We distinguish two possibilities. If $i=1$, then we have $(-d+2) a_{1} \equiv 0 \bmod n$ and, since $-d+2$ is coprime with $n$, we obtain $a_{1} \equiv 0 \bmod n$, which forces $a_{1}=n$ being $a_{1} \leq n$. Therefore, $0<b_{1} \leq a_{1}+1$ ranges over all possible residues modulo $n$ and we conclude as before. Suppose now that $i>1$. We choose $b_{i}=a_{i}+1, b_{i-1}=a_{i-1}$, and $b_{j}=a_{j}+1$ for all $j=1, \ldots, i-2$. Then, we obtain

$$
\begin{aligned}
b_{1}+\cdots+b_{i-1}+b_{i} t_{d} & \equiv a_{1}+\cdots+a_{i-1}+(-d+2) a_{i}+(i-2) \cdot 1+(-d+2) \\
& \equiv-(d-i) \bmod n .
\end{aligned}
$$

Hence, $R^{G}$ is nearly Gorenstein.

The case $d=2$ was left out from the previous proposition, but in fact twodimensional cyclic quotient singularities are always nearly Gorenstein.

Corollary 2.5 If $R=\mathbb{k} \llbracket x_{1}, x_{2} \rrbracket$ and $G$ is cyclic, then $R^{G}$ is nearly Gorenstein.

Proof Let $a_{1} \leq n$ be such that $a_{1} t_{\sigma(1)} \equiv 0 \bmod n$. Since there are no pseudoreflections in $G$, we have $\operatorname{gcd}\left(t_{\sigma(1)}, n\right)=1$ and, then, $n$ divides $a_{1}$. In particular, $a_{1}=n$. Therefore, there is a solution of the equation $b_{1} t_{\sigma(1)} \equiv-t_{\sigma(2)} \bmod n$ such that $0<b_{1} \leq n=a_{1}$ and Theorem 2.3 implies that $R^{G}$ is nearly Gorenstein.

Now, we focus on groups with small order. We recall that for $n=2$ and $d$ even the $\operatorname{ring} R^{G}$ is Gorenstein since $G \subseteq \operatorname{SL}(d, \mathbb{k})$. More generally, we prove that for $n \leq 3$ it is always nearly Gorenstein.

Corollary 2.6 Let $G$ be a cyclic group of order at most 3, then $R^{G}$ is nearly Gorenstein. 
Proof We prove only the case of order 3 , since the case when $|G|=2$ can be done in the same way. So, assuming $|G|=3$, we will prove that $R^{G}$ is nearly Gorenstein by using Theorem 2.3. Let $\left(a_{1}, \ldots, a_{i}\right)$ be positive integers such that $a_{1} t_{\sigma(1)}+\cdots+a_{i} t_{\sigma(i)} \equiv 0$ mod 3 for a permutation $\sigma$ of $\{1, \ldots, d\}$. We need to find positive integers $b_{j} \leq a_{j}+1$ such that $\sum_{j=1}^{i} b_{j} t_{\sigma(j)} \equiv-\sum_{k=i+1}^{d} t_{\sigma(k)} \bmod 3$. If $-\sum_{k=i+1}^{d} t_{\sigma(k)} \equiv 0 \bmod 3$, it is enough to set $b_{j}=a_{j}$ for every $j=1, \ldots, i$. If $-\sum_{k=i+1}^{d} t_{\sigma(k)} \equiv 1 \bmod 3$ and there exists $1 \leq p \leq i$ such that $t_{\sigma(p)} \equiv 1 \bmod 3$, then we can set $b_{p}=a_{p}+1$ and $b_{j}=a_{j}$ for $1 \leq j \leq i, j \neq p$. Assume now that $t_{\sigma(j)} \equiv 2 \bmod 3$ for every $j=1, \ldots, i$. If $i=1$, then $a_{1}$ has to be equal to 3 and we can put $b_{1}=2$, otherwise it is enough to set $b_{1}=a_{1}+1, b_{2}=a_{2}+1$ and $b_{j}=a_{j}$ for $3 \leq j \leq i$. The case $-\sum_{k=i+1}^{d} t_{\sigma(k)} \equiv 2 \bmod 3$ is analogous to the previous one.

As soon as the dimension of $R$ is bigger than 2 and the order of $G$ is greater than 3 , it is possible to find cyclic quotient singularities $R^{G}$ that are not nearly Gorenstein. In order to exhibit some examples we state a necessary condition which follows immediately from Theorem 2.3.

Remark 2.7 Let $\frac{1}{n}\left(t_{1}, \ldots, t_{d}\right)$ be nearly Gorenstein. If $\operatorname{gcd}\left(t_{\sigma(1)}, \ldots, t_{\sigma(i)}, n\right)=m>$ 1 for some $i>0$ and some permutation $\sigma$ of $\{1, \ldots, d\}$, then $t_{\sigma(i+1)}+\cdots+t_{\sigma(d)} \equiv 0$ mod $m$. Indeed, if we choose $a_{1}=n$, Theorem 2.3 implies that there exists $b_{1}$ such that $b_{1} t_{\sigma(1)} \equiv-\sum_{k=2}^{d} t_{\sigma(k)} \bmod n$. Therefore, it is enough to consider this congruence modulo $m$.

Example 2.8 (1) Let $\operatorname{gcd}\left(n, t_{1}\right)=m>2$, with $\operatorname{gcd}(m+1, n)=1$ and let $t_{1}=t_{2}=$ $\cdots=t_{d-2}$ and $t_{d-1}=t_{d}=m+1$. Therefore, $\operatorname{gcd}\left(t_{1}, \ldots, t_{d-2}, n\right)=m>1$, but $t_{d-1}+t_{d} \equiv 2 \not \equiv 0 \bmod m$. Hence, $R^{G}$ is not nearly Gorenstein by the previous remark. For instance, $\frac{1}{8}(4,5,5)=\frac{1}{8}(1,1,4)$ is not nearly Gorenstein.

(2) Let $t_{1}=1, t_{2}=n-1, t_{3}=n-2$. We have $t_{1}+t_{2} \equiv 0 \bmod n$, but there are no $0<b_{1}, b_{2} \leq 2$ such that $b_{1}-b_{2} \equiv 2 \bmod n$. Hence, Theorem 2.3 implies that $\frac{1}{n}\left(t_{1}, t_{2}, t_{3}\right)$ is not nearly Gorenstein. In particular $\frac{1}{4}(1,2,3)$ is not nearly Gorenstein. We also notice that in this case, if $n>3$ is odd, we have $\operatorname{gcd}\left(t_{\sigma(1)}, \ldots, t_{\sigma(i)}, n\right)=1$ for every $i$ and, therefore, the converse of Remark 2.7 does not hold.

Another interesting class of nearly Gorenstein quotient singularities is given by Veronese subalgebras, which are obtained when $t_{1}=t_{2}=\cdots=t_{d}=1$. See (Herzog et al. 2019, Corollary 4.8) for a proof in the positively graded case.

\section{Corollary 2.9 The Veronese subalgebras of $R$ are nearly Gorenstein.}

Proof Let $0<i<d$ and let $a_{1}, \ldots, a_{i}$ be positive integers such that $a_{1}+\cdots+a_{i}=n$. Let $k n<d \leq(k+1) n$ for some non-negative integer $k$.

Assume first that $d-i-k n \geq 0$. Then, we have $0 \leq d-i-k n \leq n-i$ that implies $i \leq n-(d-i-k n) \leq n=\sum_{j=1}^{i} a_{j}$. Therefore, there exist $b_{1}, \ldots, b_{i}$ such that $1 \leq b_{j} \leq a_{j}$ and $\sum_{j=1}^{i} b_{j} t_{\sigma(j)} \equiv n-(d-i-k n) \equiv-\sum_{k=i+1}^{d} t_{\sigma(k)} \bmod n$. 
Table 1 Cyclic quotient singularities for $d=3$ and $4 \leq n \leq 7$, and for $d=4$ and $4 \leq n \leq 6$. G means Gorenstein and NG means nearly Gorenstein

\begin{tabular}{|c|c|c|c|c|c|}
\hline Ring & $\begin{array}{l}\text { Is nearly } \\
\text { Gorenstein }\end{array}$ & Ring & $\begin{array}{l}\text { Is nearly } \\
\text { Gorenstein }\end{array}$ & Ring & $\begin{array}{l}\text { Is nearly } \\
\text { Gorenstein }\end{array}$ \\
\hline$\frac{1}{4}(1,1,1)$ & NG & $\frac{1}{4}(1,1,2)$ & G & $\frac{1}{4}(1,1,3)$ & NG \\
\hline$\frac{1}{4}(1,2,3)$ & Not NG & $\frac{1}{5}(1,1,1)$ & NG & $\frac{1}{5}(1,1,2)$ & NG \\
\hline$\frac{1}{5}(1,1,3)$ & G & $\frac{1}{5}(1,1,4)$ & NG & $\frac{1}{5}(1,2,3)$ & Not NG \\
\hline$\frac{1}{6}(1,1,1)$ & NG & $\frac{1}{6}(1,1,2)$ & NG & $\frac{1}{6}(1,1,3)$ & Not NG \\
\hline$\frac{1}{6}(1,1,4)$ & G & $\frac{1}{6}(1,1,5)$ & NG & $\frac{1}{6}(1,2,3)$ & G \\
\hline$\frac{1}{6}(1,2,5)$ & Not NG & $\frac{1}{6}(1,3,4)$ & Not NG & $\frac{1}{6}(1,3,5)$ & Not NG \\
\hline$\frac{1}{7}(1,1,1)$ & NG & $\frac{1}{7}(1,1,2)$ & NG & $\frac{1}{7}(1,1,3)$ & Not NG \\
\hline$\frac{1}{7}(1,1,4)$ & NG & $\frac{1}{7}(1,1,5)$ & G & $\frac{1}{7}(1,1,6)$ & NG \\
\hline$\frac{1}{7}(1,2,3)$ & Not NG & $\frac{1}{7}(1,2,4)$ & G & $\frac{1}{7}(1,2,5)$ & Not NG \\
\hline$\frac{1}{7}(1,2,6)$ & Not NG & $\frac{1}{4}(1,1,1,1)$ & G & $\frac{1}{4}(1,1,1,2)$ & Not NG \\
\hline$\frac{1}{4}(1,1,1,3)$ & Not NG & $\frac{1}{4}(1,1,2,2)$ & NG & $\frac{1}{4}(1,1,2,3)$ & Not NG \\
\hline$\frac{1}{4}(1,1,3,3)$ & G & $\frac{1}{4}(1,2,2,3)$ & G & $\frac{1}{5}(1,1,1,1)$ & NG \\
\hline$\frac{1}{5}(1,1,1,2)$ & G & $\frac{1}{5}(1,1,1,3)$ & NG & $\frac{1}{5}(1,1,1,4)$ & Not NG \\
\hline$\frac{1}{5}(1,1,2,2)$ & NG & $\frac{1}{5}(1,1,2,3)$ & NG & $\frac{1}{5}(1,1,2,4)$ & Not NG \\
\hline$\frac{1}{5}(1,1,3,4)$ & NG & $\frac{1}{5}(1,1,4,4)$ & G & $\frac{1}{5}(1,2,3,4)$ & G \\
\hline$\frac{1}{6}(1,1,1,1)$ & NG & $\frac{1}{6}(1,1,1,2)$ & Not NG & $\frac{1}{6}(1,1,1,3)$ & G \\
\hline$\frac{1}{6}(1,1,1,4)$ & Not NG & $\frac{1}{6}(1,1,1,5)$ & Not NG & $\frac{1}{6}(1,1,2,2)$ & G \\
\hline$\frac{1}{6}(1,1,2,3)$ & Not NG & $\frac{1}{6}(1,1,2,4)$ & NG & $\frac{1}{6}(1,1,2,5)$ & Not NG \\
\hline$\frac{1}{6}(1,1,3,3)$ & Not NG & $\frac{1}{6}(1,1,3,4)$ & Not NG & $\frac{1}{6}(1,1,3,5)$ & Not NG \\
\hline$\frac{1}{6}(1,1,4,4)$ & NG & $\frac{1}{6}(1,1,4,5)$ & Not NG & $\frac{1}{6}(1,1,5,5)$ & G \\
\hline$\frac{1}{6}(1,2,2,3)$ & Not NG & $\frac{1}{6}(1,2,2,5)$ & Not NG & $\frac{1}{6}(1,2,3,3)$ & Not NG \\
\hline$\frac{1}{6}(1,2,3,4)$ & Not NG & $\frac{1}{6}(1,2,3,5)$ & Not NG & $\frac{1}{6}(1,2,4,5)$ & G \\
\hline$\frac{1}{6}(1,3,3,4)$ & Not NG & $\frac{1}{6}(1,3,3,5)$ & G & $\frac{1}{6}(1,3,4,4)$ & G \\
\hline
\end{tabular}

Assume now that $d-i-k n<0$. It follows that $0<k n-d+i<i$ and then

$$
\begin{aligned}
\sum_{j=1}^{k n-d+i}\left(a_{j}+1\right) t_{\sigma_{j}}+\sum_{l=k n-d+i+1}^{i} a_{l} t_{\sigma_{l}} & \equiv n+k n-d+i \\
& \equiv-(d-i) \equiv-\sum_{k=i+1}^{d} t_{\sigma(k)} \bmod n
\end{aligned}
$$

Hence, the claim follows by Theorem 2.3.

In Table 1 we present an exhaustive list of non-isomorphic cyclic quotient singularities for $d=3$ and $4 \leq n \leq 7$, and for $d=4$ and $4 \leq n \leq 6$. Moreover, by 
using the numerical criterion of Theorem 2.3, we report if they are Gorenstein, nearly Gorenstein or not.

We conclude this section by recalling that the residue of a local ring is a measure of how far is a ring from being nearly Gorenstein. In the next theorem we show that we have cyclic quotient singularities of arbitrarily large residue already in dimension 3.

Theorem 2.10 Let $R=\mathbb{k} \llbracket x, y, z \rrbracket$ and let $n$ and $m$ be two coprime positive integers with $n \geq 3$ and $m<\left\lceil\frac{n}{2}\right\rceil$. Consider the group $G$ generated by $\operatorname{diag}\left(\lambda, \lambda^{m}, \lambda^{n-1}\right)$, where $\lambda$ is a primitive $n$-th root of unit in $\mathbb{k}$. Then, $\operatorname{res}\left(R^{G}\right)=m$. In particular, $R^{G}$ is nearly Gorenstein if and only if $m=1$.

Proof In order to compute $\operatorname{res}\left(R^{G}\right)=\ell_{R^{G}}\left(R^{G} / \operatorname{tr}\left(\omega_{R^{G}}\right)\right)$ we count how many monomials of the maximal ideal $\mathfrak{m}$ of $R^{G}$ are not in $\operatorname{tr}\left(\omega_{R} G\right)$. We fix $f=x y z$ and we recall that $\operatorname{tr}\left(\omega_{R^{G}}\right)=(f R)^{G}(R: Q(R) f R)^{G}$ by Proposition 2.1. Let $g=x^{a} y^{b} z^{c} \in \mathfrak{m}$. If $a, b, c>0$, then we can write $g=f x^{a-1} y^{b-1} z^{c-1} \in(f R)^{G} \subseteq \operatorname{tr}\left(\omega_{R^{G}}\right)$. If $b=c=0$, then $g=x^{a} \in \mathfrak{m}$ implies that $n$ divides $a$. Then, the condition of Lemma 2.2 is satisfied for $g$, since $b_{1}$ gives all possible residues modulo $n$ for $0<b_{1} \leq a+1$. Therefore, $x^{a} \in \operatorname{tr}\left(\omega_{R^{G}}\right)$. Similarly, one obtains that $y^{b}, z^{c} \in \operatorname{tr}\left(\omega_{R^{G}}\right)$ because $\operatorname{gcd}(m, n)=\operatorname{gcd}(n-1, n)=1$. It remains to check the monomials of the form $x^{a} y^{b}, x^{a} z^{c}, y^{b} z^{c} \in \mathfrak{m}$ with $a, b, c>0$. We use again the criterion of Lemma 2.2. If $y^{b} z^{c} \in \mathfrak{m}$, then $m b+(n-1) c \equiv 0 \bmod n$. It follows that $m b+(n-1)(c+1) \equiv n-1 \equiv-1=-t_{1} \bmod n$, therefore $y^{b} z^{c} \in \operatorname{tr}\left(\omega_{R^{G}}\right)$. If $x^{a} y^{b} \in \mathfrak{m}$, then $a+m b \equiv 0 \bmod n$ implies $(a+1)+m b \equiv 1 \equiv-(n-1)=-t_{3}$ $\bmod n$, thus $x^{a} y^{b} \in \operatorname{tr}\left(\omega_{R^{G}}\right)$. Finally, consider a monomial $x^{a} z^{c} \in \mathfrak{m}$. If $a \geq n$ or $c \geq n$, then $x^{a} y^{b} \in \operatorname{tr}\left(\omega_{R^{G}}\right)$ because $x^{n}$ and $y^{n}$ are in $\operatorname{tr}\left(\omega_{R^{G}}\right)$, therefore we may assume $a, c<n$. Since $x^{a} z^{c} \in \mathfrak{m}$, we have $a+(n-1) c \equiv 0 \bmod n$, thus $a \equiv c \bmod n$ which implies $a=c$. By Lemma 2.2, $x^{a} z^{c} \in \operatorname{tr}\left(\omega_{R^{G}}\right)$ if and only if there exist $0<b_{1}, b_{3} \leq a+1$ such that $b_{1}-b_{3} \equiv-m \bmod n$. We notice that $b_{1}-b_{3} \in\{a, a-1, \ldots,-a+1,-a\}$ and, so, there are exactly $m-1$ monomials in $\mathfrak{m}$ of the form $x^{a} z^{a}$ that do not satisfy this criterion: $x^{a} z^{a}$ with $1 \leq a \leq m-1$. Hence, $\operatorname{dim}_{R^{G} / \mathfrak{m}}\left(R^{G} / \operatorname{tr}\left(\omega_{R^{G}}\right)\right)=\operatorname{dim}_{R^{G} / \mathfrak{m}}\left(\mathfrak{m} / \operatorname{tr}\left(\omega_{R^{G}}\right)\right)+1=m$.

\section{An addendum to Ding's classification in dimension two}

Let $\mathbb{C}$ be the field of complex numbers and set $R=\mathbb{C} \llbracket u, v \rrbracket$. In his paper (Ding 1993), Ding classified nearly Gorenstein quotient singularities $R^{G}$, where $G$ is a finite small subgroup of GL(2, $\mathbb{C})$. His result relies on the well-known classification of such subgroups which goes back to Klein (1884) (see also Beauville 2010; Brieskorn 1968; Riemenschneider 1977). However, a nearly Gorenstein quotient singularity was left out of his classification. We are going to describe it.

We consider the octahedral group $\mathbb{O}_{11}$ generated by matrices

$$
\phi=\left(\begin{array}{ll}
\lambda & 0 \\
0 & \lambda
\end{array}\right), \psi=\left(\begin{array}{cc}
\zeta & 0 \\
0 & \zeta^{-1}
\end{array}\right), \tau=\left(\begin{array}{ll}
0 & i \\
i & 0
\end{array}\right), \eta=\frac{1}{\sqrt{2}}\left(\begin{array}{ll}
\zeta & \zeta^{3} \\
\zeta & \zeta^{7}
\end{array}\right),
$$


where $\lambda$ and $\zeta$ are primitive roots of unity in $\mathbb{C}$ of orders 22 and 8 respectively. In other words, $\mathbb{O}_{11}$ is the extension of the binary octahedral subgroup of $\operatorname{SL}(2, \mathbb{C})$ of order 48 generated by $\psi, \tau, \eta$ with the cyclic group of order 22 generated by $\phi$.

Proposition 3.1 The quotient singularity $R^{\mathbb{O}_{11}}$ is nearly Gorenstein.

Proof We consider the polynomials

$$
\begin{aligned}
& g_{1}=\left(u^{5} v-u v^{5}\right)^{2}, \\
& g_{2}=u v\left(u^{4}-v^{4}\right)\left(u^{12}-33 u^{8} v^{4}-33 u^{4} v^{8}+v^{12}\right), \\
& g_{3}=u^{8}+14 u^{4} v^{4}+v^{8},
\end{aligned}
$$

which are generating invariants for the Gorenstein singularity $E_{7}$ (see e.g. Leuschke and Wiegand 2012, Sect. 6.16) and in particular invariants for the action of $\psi, \tau$, and $\eta$. By Riemenschneider (1977, Satz 6), a minimal set of generators for the maximal ideal of $R^{\mathbb{O}_{11}}$ is given by

$$
z_{1}=g_{3}^{11}, z_{2}=g_{1} g_{3}^{4}, z_{3}=g_{1}^{4} g_{2}, z_{4}=g_{2} g_{3}^{6}, z_{5}=g_{1}^{3} g_{3}, z_{6}=g_{1}^{2} g_{2} g_{3}^{3} \text {. }
$$

Now, we consider the polynomial $f=g_{3}^{3}$. It is invariant for the action of the matrices $\psi, \tau, \eta \in \operatorname{SL}(2, \mathbb{C})$, and $\phi(f)=\lambda^{24} f=\operatorname{det}(\phi) f$. Therefore, $f$ is a $\mathbb{O}_{11 \text {-canonical }}$ element and, thus, $\omega_{R_{11}}=(f R)^{\mathbb{O}_{11}}$ is a canonical ideal of $R^{\mathbb{O}_{11}}$ by Theorem 1.4.

We have that $z_{1}, z_{2}, z_{4}$ and $z_{6}$ are multiples of $f$, then they are in $\operatorname{tr}\left(\omega_{R^{\mathbb{Q}_{11}}}\right)$. Moreover, the fact

$$
\frac{z_{3}}{z_{6}}=\frac{z_{5}}{z_{2}}=\frac{g_{1}^{2}}{g_{3}^{3}} \in\left(R^{\mathbb{O}_{11}}:{ }_{Q\left(R^{\mathbb{O}_{11}}\right)}\left(f R \cap R^{\mathbb{O}_{11}}\right)\right)
$$

implies that $z_{3}=z_{6} \frac{z_{3}}{z_{6}}, z_{5}=z_{2} \frac{z_{5}}{z_{2}} \in \operatorname{tr}\left(\omega_{R^{\mathbb{O}_{11}}}\right)$ as well. Hence, $R^{\mathbb{O}_{11}}$ is nearly Gorenstein.

We also point out that Ding erroneously included cyclic quotient singularities $\frac{1}{n}(1, n-1)$ in his list of nearly Gorenstein not Gorenstein quotient singularities. Indeed, it is well known that $\frac{1}{n}(1, n-1)$ is Gorenstein.

Acknowledgements This work began at the Institute of Mathematics of the University of Barcelona (IMUB). The authors would like to express their gratitude to the IMUB for providing a fruitful work environment.

Funding Open access funding provided by University of Neuchâtel.

Open Access This article is licensed under a Creative Commons Attribution 4.0 International License, which permits use, sharing, adaptation, distribution and reproduction in any medium or format, as long as you give appropriate credit to the original author(s) and the source, provide a link to the Creative Commons licence, and indicate if changes were made. The images or other third party material in this article are included in the article's Creative Commons licence, unless indicated otherwise in a credit line to the material. If material is not included in the article's Creative Commons licence and your intended use is not permitted by statutory regulation or exceeds the permitted use, you will need to obtain permission directly from the copyright holder. To view a copy of this licence, visit http://creativecommons.org/licenses/by/4.0/. 


\section{References}

Barucci, V., Fröberg, R.: One-dimensional almost Gorenstein rings. J. Algebra 188(2), 418-442 (1997)

Bass, H.: On the ubiquity of Gorenstein rings. Math. Z. 82, 8-28 (1963)

Beauville, A.: Finite subgroups of $P G L_{2}(K)$, Vector bundles and complex geometry, 23-29, Contemp. Math. 522, Am. Math. Soc., Providence, RI, (2010)

Brieskorn, E.: Rationale Singularitäten komplexer Flächen. Invent. Math. 4(5), 336-358 (1968)

Bruns, W., Herzog, J.: Cohen-Macaulay rings. Revised edition, Cambridge Studies in Advanced Mathematics, vol. 39, Cambridge University Press, (1998)

Dao, H., Kobayashi, T. , Takahashi, R.: Trace of canonical modules, annihilator of Ext, and classes of rings close to being Gorenstein, arXiv:2005.02263

Ding, S.: A note on the index of Cohen-Macaulay local rings. Commun. Algebra 21(1), 53-71 (1993)

Campbell, H.E.A., Wehlau, D.: Modular invariant theory, encyclopaedia of mathematical sciences vol. 139, Springer, Berlin Heidelberg, (2011)

Endo, N., Goto, S., Isobe, R.: Almost Gorenstein rings arising from fiber products, Can. Math. Bull. (to appear)

Goto, S., Matsuoka, N., Phuong, T.T.: Almost Gorenstein rings. J. Algebra 379, 355-381 (2013)

Goto, S., Takahashi, R., Taniguchi, N.: Almost Gorenstein rings-towards a theory of higher dimension. J. Pure Appl. Algebra 219(7), 2666-2712 (2015)

Goto, S., Takahashi, R., Taniguchi, N.: Ulrich ideals and almost Gorenstein rings. Proc. Am. Math. Soc. 144, 2811-2823 (2016)

Herzog, J., Jafari, R., Stamate, D. I.: Ulrich elements in normal simplicial affine semigroups arXiv: 1909.06846

Herzog, J., Hibi, T., Stamate, D.I.: The trace of the canonical module. Isr. J. Math. 233, 133-165 (2019)

Hochster, M., Eagon, J.A.: Cohen-Macaulay rings, invariant theory, and the generic perfection of determinantal loci. Am. J. Math. 93, 1020-1058 (1971)

Huneke, C.: Hyman Bass and ubiquity: Gorenstein rings. Algebra, K-theory, groups, and education (New York, 1997), 55-78, Contemp. Math. 243, Am. Math. Soc., Providence, RI, (1999)

Huneke, C., Vraciu, A.: Rings that are almost Gorenstein. Pacific J. Math. 225, 85-102 (2006)

Kemper, G.: On the Cohen-Macaulay Property of modular invariant rings. J. Algebra 215(1), 330-351 (1999)

Klein, F.: Vorlesungen über das Ikosaeder und die Auflösung der Gleichungen vom fünften Grade. Teubner, Leipzig (1884)

Kobayashi, T.: Local rings with self-dual maximal ideal arXiv:1812.10341

Kreuzer, M., Linh, T.N.K., Long, N.: The dedekind different of a Cayley-Bacharach scheme. J. Algebra Appl. 18(2), 1950027 (2019)

Kumashiro, S.: Almost reduction number of canonical ideals arXiv:2007.00377

Leuschke, G., Wiegand, R.: Cohen-Macaulay representations. Mathematical surveys and monographs 181. American Mathematical Society, Providence (2012)

Moscariello, A., Strazzanti, F.: Nearly Gorenstein vs almost Gorenstein affine monomial curves. Mediterr. J. Math. arXiv:2003.05391

Prill, D.: Local classification of quotients of complex manifolds by discontinuous groups. Duke Math. J. 34, 375-386 (1967)

Rahimi, M.: Rings with canonical reductions. Bull. Iranian Math. Soc. (to appear)

Riemenschneider, O.: Die Invarianten der endlichen Untergruppen von $G L(2, \mathbb{C})$. Math. Z. 153, 37-50 (1977)

Singh, B.: Invariants of finite groups acting on local unique factorization domains. J. Indian Math. Soc. 34, 31-38 (1970)

Stanley, R.P.: Hilbert functions of graded algebras. Adv. Math. 28(1), 57-83 (1978)

Striuli, J., Vraciu, A.: Some homological properties of almost Gorenstein rings, Commutative algebra and its connections to geometry, 201-215, Contemp. Math., 555, Am. Math. Soc., Providence, RI, (2011)

Taniguchi, N.: On the almost Gorenstein property of determinantal rings. Comm. Algebra 46, 1165-1178 (2018)

Watanabe, K.: Certain invariant subrings are Gorenstein I. Osaka J. Math. 11(1), 1-8 (1974)

Watanabe, K.: Certain invariant subrings are Gorenstein II. Osaka J. Math. 11(2), 379-388 (1974) 
Publisher's Note Springer Nature remains neutral with regard to jurisdictional claims in published maps and institutional affiliations. 